University of Windsor

Scholarship at UWindsor

1992

\title{
Stationary solutions for an electron in an intense laser field. II. Multimode case
}

D. S. Guo

Gordon W. F. Drake

University of Windsor

Follow this and additional works at: https://scholar.uwindsor.ca/physicspub

Part of the Physics Commons

\section{Recommended Citation}

Guo, D. S. and Drake, Gordon W. F.. (1992). Stationary solutions for an electron in an intense laser field. II. Multimode case. Journal of Physics A: Mathematical and General, 25 (20), 5377-5394.

https://scholar.uwindsor.ca/physicspub/32

This Article is brought to you for free and open access by the Department of Physics at Scholarship at UWindsor. It has been accepted for inclusion in Physics Publications by an authorized administrator of Scholarship at UWindsor. For more information, please contact scholarship@uwindsor.ca. 
Stationary solutions for an electron in an intense laser field. II. Multimode case

This article has been downloaded from IOPscience. Please scroll down to see the full text article.

1992 J. Phys. A: Math. Gen. 255377

(http://iopscience.iop.org/0305-4470/25/20/018)

View the table of contents for this issue, or go to the journal homepage for more

Download details:

IP Address: 137.207.184.30

The article was downloaded on 26/04/2013 at 02:39

Please note that terms and conditions apply. 


\title{
Stationary solutions for an electron in an intense laser field:
}

\section{II. multimode case}

\author{
Dong-Sheng Guo and G W F Drake \\ Department of Physics, University of Windsor, Windsor, Ontario, Canada N9B 3P4
}

Received 24 February 1992, in final form 17 June 1992

\begin{abstract}
The Schrödinger equation for an electron and a multimode photon field with interactions is solved in the large-photon-number limit by using an 'integration' method. A graphical technique different from Feynman's is developed to represent the terms in the solution. By this graphical technique, all interactions between the electron and the multimode photon field are evaluated to any arbitrary order according to the number of transferred photons. The graphical tectniquie allows one easily to write down the wavefunctions for an electron interacting with a strong photon field which contains an arbitrary number of photon modes. The two-mode case is discussed in detail as an example. Some interesting physical questions arising from the solutions are briefly discussed. As a simple application, a direct generalization of the Keldysh-Faisal-Reiss formula for the transition rate of multiphoton ionization, is given in the case where two different laser beams are applied.
\end{abstract}

\section{Introduction}

This paper is a continuation of an earlier work (Guo and Drake 1992a, to be referred to as I) in which we obtained stationary solutions for an electron in an intense singlemode laser field. Here we generalize the solutions to the multimode case. We begin by developing an 'integration' method for the single-mode case which can readily be generalized to fields with any number of modes. The solution to the Schodinger equation is obtained directly by neglecting terms which become infinitesimal in the large-photon-number limit. In this regard, the method differs from that in I, where we took the large-photon-number limit of the exact quantized-field solution. The two

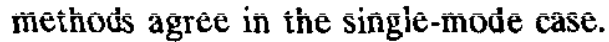

A general discussion and historical background for the single-mode case is given in I. There are many reasons for extending these solutions to the multimode case. For example, recent experiments on multiphoton ionization in standing electromagnetic waves (Bucksbaum et al 1988) can be regarded as a two-mode problem with counterpropagating waves. This has been treated separately in a previous paper (Guo and Drake 1992b). Even if the initial laser beam contains only one mode, scattering p processes can produce additional modes. Also, electrons may absorb from one mode and emit into another (mode conversion). Finally it may be useful to Fourier transform time-dependent interactions into an effective multimode problem.

During the last two decades, the classical solutions of Volkov (1935) and Gordon (1926) have been treated as quantized fields by several authors, as discussed in I. 
All these solutions are limited to a single frequency, polarization and direction of propagation. The present solutions have the following four features.

(1) The modes can propagate in arbitrary directions.

(2) All modes can have arbitrary elliptical polarizations.

(3) The present solutions are for the large-photon-number limit, but in the quantized-field version, thus making it possible to describe absorption and emission processes with definite transferred photon numbers. They also enable us to treat the electron and photons as an isolated system, so that the wavefunctions for the electron and photons are energy eigenfunctions of the Hamiltonian.

(4) The present solutions are non-relativistic for the electron, but there is no long-wavelength approximation for the photons; i.e. retardation is included in the photon vector-potential, and hence in the photon part of the wavefunction. This feature is particularly advantageous for treating strong radiation fields, in contrast with earlier non-relativistic semiclassical approaches that are mostly in the dipole approximation or in the long-wavelength approximation (Keldysh 1964, Faisal 1973, Reiss 1980, Rosenberg 1982, Chu and Cooper 1985, Ehlotzky 1985), where the lightcone directions are deformed, limiting their range of application.

The paper is organized as follows. In section 2 we develop the 'integration method' and present a graphical representation which allows the solutions to be easily written down. Section 3 discusses in detail the two-mode case as an example, and section 4 gives the generalization to an arbitrary number of modes. Finally, section 5 gives a brief discussion of some potential applications.

\section{The 'integration' method}

To develop the 'integration' method for solving the Schrödinger equation for an electron interacting with a multimode photon field, we will treat the single-mode case first. Since the solutions in the single-mode case have been obtained and discussed in detail previously (Guo et al 1989, Guo 1990, and I), here we will concentrate on the solving technique for later multimode generalizations. In the present paper, we use units with $\hbar=c=1$, and $e=-|e|$.

The Hamiltonian for a non-relativistic electron in a single-mode quantized radiation field can be obtained from the minimum-coupling principle as

$$
H=\frac{(-\mathrm{i} \nabla)^{2}}{2 m_{e}}-\frac{e}{2 m_{e}}[(-\mathrm{i} \nabla) \cdot A(-k \cdot r)+A(-k \cdot r) \cdot(-\mathrm{i} \nabla)]+\frac{e^{2} A^{2}(-k \cdot r)}{2 m_{e}}+\omega N_{a}
$$

where

$$
A(-k \cdot r)=g\left(\epsilon \mathrm{e}^{i k \cdot r} a+\epsilon^{*} \mathrm{e}^{-i k \cdot r} a^{\dagger}\right)
$$

and $g=\left(2 V_{\gamma} \omega\right)^{-1 / 2}, V_{\gamma}$ being the normalization volume of the photon field. $N_{a}$ is the photon number operator:

$$
N_{a}=\frac{1}{2}\left(a a^{\dagger}+a^{\dagger} a\right)
$$


The polarization vectors $\epsilon$ and $\epsilon^{*}$ are defined by

$$
\begin{aligned}
& \epsilon=\left[\epsilon_{x} \cos (\xi / 2)+\mathrm{i} \epsilon_{y} \sin (\xi / 2)\right] \mathrm{e}^{\mathrm{i} \Theta / 2} \\
& \epsilon^{*}=\left[\epsilon_{x} \cos (\xi / 2)-\mathrm{i} \epsilon_{y} \sin (\xi / 2)\right] \mathrm{e}^{-\mathrm{i} \Theta / 2}
\end{aligned}
$$

and satisfiy

$$
\epsilon \cdot \epsilon^{*}=1 \quad \epsilon \cdot \epsilon=\cos \xi \mathrm{e}^{\mathrm{i} \Theta} \quad \epsilon^{*} \cdot \epsilon^{*}=\cos \xi \mathrm{e}^{-\mathrm{i} \Theta} .
$$

The angle $\xi$ monitors the degree of polarization, such that $\xi=\pi / 2$ corresponds to circular polarization and $\xi=0$ to linear polarization. The phase angle $\Theta$ is introduced to characterize the initial phase value of the simple harmonic oscillator (Guo 1990). With this phase, a full squeezed light transformation (Loudon and Knight 1987) can be fulfilled in the solving process. In multimode cases, the relative value of this phase for each mode will be important.

The Schrödinger equation to be solved is the eigenvalue equation

$$
H \Psi(r)=\mathcal{E} \Psi(r) .
$$

Writing $\Psi(\boldsymbol{r})$ in the form

$$
\Psi(r)=\mathrm{e}^{\mathrm{i} p \cdot r-\mathrm{i} k \cdot r N_{\mathrm{a}}} \phi
$$

and defining

$$
\boldsymbol{A}=\mathrm{e}^{\mathrm{i} \boldsymbol{k} \cdot \boldsymbol{r} N_{a}} \boldsymbol{A}(-\boldsymbol{k} \cdot \boldsymbol{r}) \mathrm{e}^{-\mathrm{i} \boldsymbol{k} \cdot \boldsymbol{r} N_{c}}=g\left(\epsilon a+\epsilon^{\prime \prime} a^{\dagger}\right)
$$

we show in I that the Schrödinger equation (6) reduces to the coordinate-independent form

$$
\left(\frac{\boldsymbol{P}^{2}}{2 m_{e}}-\frac{e}{m_{e}} \boldsymbol{P} \cdot \boldsymbol{A}+\frac{e^{2} \boldsymbol{A}^{2}}{2 m_{e}}+\omega N_{a}\right) \phi=\mathcal{E} \phi
$$

Where

$$
P=p-\kappa k
$$

and, as in $I, \kappa$ is a $c$-number determined by the requirement that the effect of the operator $\boldsymbol{k} N_{a}$ can be replaced by $\kappa \boldsymbol{k}$ in the non-relativistic limit, with $\kappa$ to be determined later (see (30) below). A detailed justification is given in I. Then (9) is a solvable equation in quantum optics (Loudon and Knight 1987).

The 'integration' method to follow makes it possible to obtain the solutions in the large-photon-number limit directly, without first going through the exact quantumfield solution. Since the method does not depend on the number of modes, once the single-mode case is solved by using the method, it can readily be generalized for multimode cases.

We start with (9), rewritten as

$$
\begin{aligned}
& \left(\frac{\boldsymbol{P}^{2}}{2 m_{e}}+H_{\gamma}^{\prime}+V^{\prime}\right) \phi=\mathcal{E} \phi \quad H_{\gamma}^{\prime}=\left(\omega+e^{2} g^{2} / m_{e}\right) N_{a} \\
& V^{\prime}=-\frac{e g}{m_{e}}\left(\boldsymbol{P} \cdot \epsilon a+\boldsymbol{P} \cdot \epsilon^{*} a^{\dagger}\right)+\frac{e^{2} g^{2}}{2 m_{e}}(\cos \xi)\left(\mathrm{e}^{\mathrm{i} \Theta} a^{2}+\mathrm{e}^{-i \Theta_{a} \dagger 2}\right) .
\end{aligned}
$$


The operator $H_{\gamma}^{\prime}$ is identified as the new free-photon-energy term with the frequency shift $e^{2} g^{2} / m_{e}$ as a contribution due to the $A^{2}$ term, while the operator $V^{\prime}$ is identified as the new interaction term.

Now any two operators $O$ and $F$ formly satisfy the relation

$e^{F} O e^{-F}=O+[F, O]+\frac{1}{2 !}[F,[F, O]]+\frac{1}{3 !}[F,[F,[F, O]]]+\cdots$.

If $F$ is an infinitesimal operator, we have

$$
e^{F} O e^{-F}=O+[F, O]
$$

a formula that we will use extensively.

For example, if $O$ is $a$ or $a^{\dagger}$, we have

$$
d=e^{F} a e^{-F}=a+[F, a] \quad d^{\dagger}=e^{F} a^{\dagger} e^{-F}=a^{\dagger}+\left[F, a^{\dagger}\right]
$$

and

$$
F^{\dagger}=-F
$$

to guarantee that $d^{\dagger}$ is a hermitian conjugate of $d$, and

$$
\left[d, d^{\dagger}\right]=\left[a, a^{\dagger}\right]=I \text {. }
$$

To find a transformation which eliminates the new interaction term, we introduce the following concepts. For an operator $O$, we define its 'derivative' or the result of 'differentiation' as

$$
\dot{O}=\left[H_{\gamma}^{\prime}, O\right]
$$

and call $O$ an 'integral' or the result of 'integration' of $\dot{O}$, which we can write (but for an arbitrary constant)

$$
O=\int \dot{O} .
$$

According to this definition, we have the following table for 'differentiation' and 'integration':

$$
\begin{aligned}
& \dot{a}=\left[H_{\gamma}^{\prime}, a\right]=-\left(\omega+e^{2} g^{2} / m_{e}\right) a \quad \dot{a}^{\dagger}=\left[H_{\gamma}^{\prime}, a^{\dagger}\right]=\left(\omega+e^{2} g^{2} / m_{e}\right) a^{\dagger} \\
& \left(\dot{a}^{2}\right)=\left[H_{\gamma}^{\prime}, a^{2}\right]=-2\left(\omega+e^{2} g^{2} / m_{e}\right) a^{2} \\
& \left(\dot{a}^{\dagger 2}\right)=\left[H_{\gamma}^{\prime}, \tilde{a}^{\dagger 2}\right]=2\left(\omega+e^{2} \tilde{g}^{2} / m_{e}\right) a^{\dagger 2} \\
& \int a=-m_{e}\left(m_{e} \omega+e^{2} g^{2}\right)^{-1} a \quad \int a^{\dagger}=m_{e}\left(m_{e} \omega+e^{2} g^{2}\right)^{-1} a^{\dagger} \\
& \int a^{2}=-m_{e}\left[2\left(m_{e} \omega+e^{2} g^{2}\right)\right]^{-1} a^{2} \quad \int a^{\dagger 2}=m_{e}\left[2\left(m_{e} \omega+e^{2} g^{2}\right)\right]^{-1} a^{\dagger 2} .
\end{aligned}
$$


The constants omitted in the 'integrations' only affect the final wavefunctions by an arbitrary normalization volume and an arbitrary phase factor which can always be inserted once the final solution is obtained.

The properties of the operations - and $\int$ include those of linearity and the product differentiation rule $(\dot{A B})=\dot{A} B+A \dot{B}$.

The method of solving the wave equation (11) is based on finding an infinitesimal operator $\bar{F}$ such that $\left[\bar{F}, \bar{H}_{\gamma}^{j}\right]$ canceis the $\bar{V}^{\prime}$ term when (11) is transformed by $e^{F}$ to

$$
\left(\frac{\boldsymbol{P}^{2}}{2 m_{e}}+e^{F} H_{\gamma}^{\prime} e^{-F}+e^{F} V^{\prime} e^{-F}\right)\left(e^{F} \phi\right)=\mathcal{E}\left(e^{F} \phi\right)
$$

We write

$$
e^{F} H_{\gamma}^{\prime} e^{-F}=H_{\gamma}^{\prime}+\left[F H_{\gamma}^{\prime}\right] \quad e^{F} V^{\prime} e^{-F}=V^{\prime}
$$

and note that $V^{\prime}$ itself is infinitesimal when $g \rightarrow 0$. Hence, if we set

$$
\dot{F}=\left[H_{\gamma}^{\prime}, F\right]=V^{\prime}
$$

the interaction term is eliminated in (20):

$$
\left(\frac{P^{2}}{2 m_{e}}+H_{\gamma}^{\prime}\right)\left(e^{F} \phi\right)=\mathcal{E}\left(e^{F} \phi\right) .
$$

The operator $F$ can be found by 'integration':

$$
F=\int V^{\prime}=\frac{e g}{m_{e} \omega+e^{2} g^{2}}\left(\boldsymbol{P} \cdot \boldsymbol{\epsilon} a-\boldsymbol{P} \cdot \epsilon^{*} a^{\dagger}\right)+\frac{e^{2} g^{2} \cos \xi}{4\left(m_{e} \omega+e^{2} g^{2}\right)}\left(\mathrm{e}^{-\mathrm{i} \Theta} a^{\dagger 2}-\mathrm{e}^{\mathrm{i} \Theta} a^{2}\right)
$$

which satisfies the relation (15). Omitting an arbitrary normalization constant, we can set

$$
e^{F} \phi=|n\rangle \quad \phi=e^{-F}|n\rangle .
$$

Using the definition (11) for $H_{\gamma}^{\prime}$, the energy eigenvalue in (23) is then found to be

$$
\mathcal{E}=\frac{\boldsymbol{P}^{2}}{2 m_{e}}+\left(n+\frac{1}{2}\right) \omega+e^{2} g^{2}\left(n+\frac{1}{2}\right) / m_{e} .
$$

If we define

$$
E=\frac{P^{2}}{2 m_{e}}
$$

we see that (27) is just the on-mass-shell condition for a non-relativistic electron with 4-momentum $\left(E+m_{e}, P\right)$. Thus we have

$$
\mathcal{E}+m_{e}=\left(E+m_{e}\right)+\kappa^{\prime} \omega
$$


where the constant $\kappa^{\prime}$ is defined by

$$
\kappa^{\prime}=\left(n+\frac{1}{2}\right)+e^{2} g^{2}\left(n+\frac{1}{2}\right) /\left(m_{e} \omega\right) .
$$

The electron is described non-relativistically, its velocity being much less than that of light, but retardation is included for the photons. Comparing (28) and (12) and using the fact that $\left(\mathcal{E}+m_{e}, \boldsymbol{p}\right),\left(E+m_{e}, \boldsymbol{P}\right)$ and $(\omega, \boldsymbol{k})$ are Lorentz 4-vectors, it is follows that

$$
\kappa=\kappa^{\prime}=\left(n+\frac{1}{2}\right)+e^{2} g^{2}\left(n+\frac{1}{2}\right) /\left(m_{e} \omega\right)
$$

which thereby fixes the value of $\kappa$. We can also define the important parameter $z$

$$
z=e^{2} g^{2}\left(n+\frac{1}{2}\right) /\left(m_{e} \omega\right)
$$

with the interpretation that $z \omega$ is the interaction energy.

If the radiation field is strong, the photon number becomes very large and the field takes on semiclassical characteristics. As in earlier work (Guo and Aberg 1988), we let

$$
g \sqrt{n} \rightarrow \Lambda \quad n \rightarrow \infty \quad g \rightarrow 0
$$

where $\Lambda$ is the amplitude of the classical field. The present formalism remains valid for weak fields if the photon normalization volume tends to infinity, because we have $g=\left(2 V_{\gamma} \omega\right)^{-1 / 2}$ and the classical amplitude $\Lambda$ of the field is finite, not infinitesimal. We will call the limit (32) the large-photon-number limit.

It will be seen later that all four terms in (24) make finite contributions to matrix elements in the large-photon-number limit. For the moment, we assume that this is the case. All commutators of the four terms will then be zero or tend to vanish in the limiting process, in view of the relation $\left[a, a^{\dagger}\right]=I$. To see this more clearly, write the terms in (24) in the form $\delta_{k_{1}} a^{\dagger k_{1}}$ and $\delta_{k_{2}}^{*} a^{k_{2}}$, where the $\delta s$ are constants and each $k_{i},(i=1,2)$ can have the values 1 or 2 . Now suppose that in the largephoton-number limit

$$
\delta_{k_{i}} n^{\frac{1}{2} k_{i}} \rightarrow C_{i} \quad\left(k_{i}=1,2\right) \quad(n \rightarrow \infty, g \rightarrow 0) .
$$

Then the matrix elements of the commutators are

$$
\begin{aligned}
&\left\langle m\left|\left[\delta_{k_{1}} a^{\dagger k_{2}}, \delta_{k_{2}}^{*} a^{k_{2}}\right]\right| n\right\rangle \rightarrow \delta_{k_{1}} \delta_{k_{2}}^{*} k_{1} k_{2} n^{\frac{1}{2}\left(k_{1}-1\right)} n^{\frac{1}{2}\left(k_{2}-1\right)} \delta_{n-k_{2}, m-k_{1}} \\
& \rightarrow k_{1} k_{2} C_{1} C_{2}^{*} \delta_{n-k_{2}, m-k_{1}} n^{-1} \rightarrow 0 \quad(n, m \rightarrow \infty, g \rightarrow 0) .
\end{aligned}
$$

The above remains true even if the $k_{i}$ are greater than 2 , as may occur in nonlinear quantum optics.

Another formula required to determine the matrix elements in the large-photonnumber limit is

$$
\begin{gathered}
\left\langle m\left|\exp \left(\delta_{k} a^{\dagger k}-\delta_{k} a^{k}\right)\right| n\right\rangle \rightarrow \sum_{q=-\infty}^{\infty} J_{-q}\left(\zeta_{k}\right) \exp \left(-\mathrm{i} q \phi_{k}\right) \delta_{m-n, k q} \\
(n \rightarrow \infty, m \rightarrow \infty, g \rightarrow 0)
\end{gathered}
$$


The proof is straightforward. The $\zeta_{k}$ and $\phi_{k}$ are determined by the conditions $\delta_{k} n^{\frac{1}{2} k} \rightarrow-\frac{1}{2} \zeta_{k} \mathrm{e}^{-\mathrm{i} \phi_{k}} \quad \zeta_{k}=\lim _{n \rightarrow \infty, g \rightarrow 0} 2\left|\delta_{k} n^{\frac{1}{2} k}\right| \quad \phi_{k}=-\arg \left(-\delta_{k} n^{\frac{1}{2} k}\right)$.

Using the property $\sum_{l}|l\rangle\langle l|=I$, we can write the wavefunction $\phi$ (cf (20) and (25)) as

$$
\phi=\sum_{l}|l\rangle\left\langle l\left|e^{-F}\right| n\right\rangle
$$

In order to evaluate the matrix element $\left\langle l\left|e^{-F}\right| n\right\rangle$ in the large-photon-number limit, we separate $F$ into two parts:

$$
\begin{aligned}
& F=F_{1}+F_{2} \\
& F_{1}=\frac{e g}{m_{e} \omega+e^{2} g^{2}}\left(P \cdot \epsilon a-P \cdot \epsilon^{*} a^{\dagger}\right) \\
& F_{2}=\frac{e^{2} g^{2} \cos \xi}{4\left(m_{e} \omega+e^{2} g^{2}\right)}\left(\mathrm{e}^{-\mathrm{i} \Theta} a^{\dagger 2}-\mathrm{e}^{\mathrm{i} \Theta} a^{2}\right) .
\end{aligned}
$$

Thus we have

$$
\left\langle l\left|e^{-F}\right| n\right\rangle=\sum_{m}\left\langle l\left|e^{-F_{1}}\right| m\right\rangle\left\langle m\left|e^{-F_{2}}\right| n\right\rangle
$$

whence we can evaluate $\left\langle l\left|e^{-F_{1}}\right| m\right\rangle$ and $\left\langle m\left|e^{-F_{2}}\right| n\right\rangle$ individually.

First we set $k=1$ in (35). Thus we have

$\left\langle l\left|e^{-F_{1}}\right| m\right\rangle=\left\langle l\left|\exp \left(\delta_{1} a^{\dagger}-\delta_{1}^{*} a\right)\right| m\right\rangle \rightarrow \sum_{q=-\infty}^{\infty} J_{-q}\left(\zeta_{1}\right) \exp \left(-\mathrm{i} q \phi_{1}\right) \delta_{l-m, q}$

where the limiting form is from Guo and Âberg (1988) (see also I). From (36) and (38) we have

$$
\begin{aligned}
& \delta_{1}=\frac{e g}{m_{e} \omega+e^{2} g^{2}} \boldsymbol{P} \cdot \boldsymbol{\epsilon}^{*} \quad \zeta_{1}=\frac{2|e| \Lambda}{m_{e} \omega}|\boldsymbol{P} \cdot \boldsymbol{\epsilon}| \\
& \phi_{1}=\tan ^{-1}\left[\left(P_{y} / P_{x}\right) \tan (\xi / 2)\right]+\frac{1}{2} \Theta .
\end{aligned}
$$

By setting $k=2$ in (35), we have

$$
\begin{aligned}
\left\langle m\left|e^{-F_{2}}\right| n\right\rangle & =\left\langle m\left|\exp \left(\delta_{2} a^{\dagger 2}-\delta_{2}^{*} a^{2}\right)\right| n\right\rangle \\
& \rightarrow \sum_{q=-\infty}^{\infty} J_{-q}\left(\zeta_{2}\right) \exp \left(-\mathrm{i} q \phi_{2}\right) \delta_{m-n, 2 q} .
\end{aligned}
$$


The arguments are found as before:

$$
\delta_{2}=\frac{1}{2} \chi \mathrm{e}^{-\mathrm{i} \Theta} \quad \zeta_{2}=\frac{1}{2} z \cos \xi \quad \phi_{2}=\Theta
$$

where $\chi=-e^{2} g^{2} \cos \xi / 4\left(m_{e} \omega+e^{2} g^{2}\right)$ and $z=e^{2} \Lambda^{2} / m_{e} \omega$.

Combining these results for $k=1$ and $k=2$, we obtain

$$
\left\langle l\left|e^{-F}\right| n\right\rangle \rightarrow \sum_{j} \mathcal{J}_{j}\left(\zeta_{1}, \zeta_{2}, \phi_{\xi}\right)^{*} \mathrm{e}^{-\mathrm{i} j\left(\phi_{\ell}+\frac{\theta}{2}\right)} \delta_{j, l-n}
$$

and

$$
\phi=\sum_{l}|l\rangle\left\langle l\left|e^{-F}\right| n\right\rangle=\sum_{j=-n}^{\infty} \mathcal{J}_{j}\left(\zeta_{1}, \zeta_{2}, \phi_{\xi}\right)^{*} \mathrm{e}^{-\mathrm{i} j\left(\phi_{\varepsilon}+\frac{\Theta}{2}\right)}|n+j\rangle .
$$

The results for the matrix elements can symbolically be expressed by diagrams. We define the following rules:

(i) A wiggly line as in figure $1(a)$ denotes a multiphoton state $|n\rangle$ with a large photon number $n$. The orthogonality relation is symbolized by figure $1(b)$. For an internal line, the photon number is to be summed over all number states $|n\rangle$.

(a)

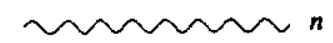

(b)

$m \sim n=\delta_{m, n}$

(c)

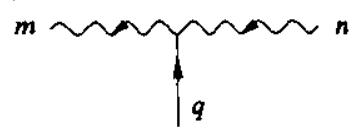

(d)

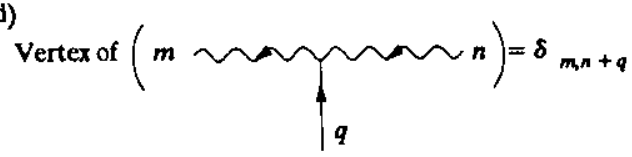

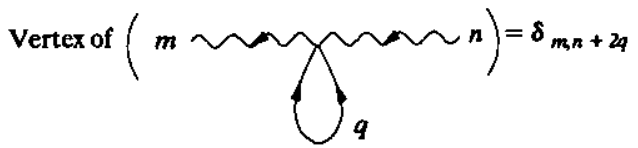

Figure 1. Definitions of the diagrammatic representation of matrix elements.

(ii) A smooth line denotes a Bessel function multiplied by a phase factor:

$$
q q=J_{-q}\left(\zeta_{1}\right) \mathrm{e}^{-\mathrm{i} q \phi_{1}}
$$


The integer $q$ is the transferred-photon number. It is to be considered a dummy variable and is to be summed from $-\infty$ to $\infty$. The meaning of the arrow is stated in the next rule.

(iii) A vertex as shown in figure $1(c)$ connects photon number states $|n\rangle$ and $|m\rangle$ with a Bessel function multiplied by a phase factor. Balance of photon numbers is required at the vertices, as indicated in figure $1(d)$. The sum of the photon numbers of lines with inward arrows equals the sum of the photon numbers of lines with outward arrows.

By these rules, (40) can be expressed graphically as shown in figure $2(a)$, and (42), as shown in figure $2(b)$. To write the matrix element $\left\langle l\left|e^{-F}\right| n\right\rangle$, we can simply connect these two diagrams (figure $2(c)$ ). It is easy to construct a proof as in figure $2(d)$; there is no need to write down the dummy variables, such as $m, q_{1}$, and $q_{2}$.

(a)

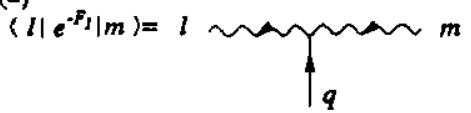

(b)

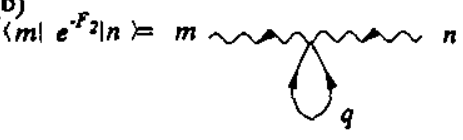

(c)

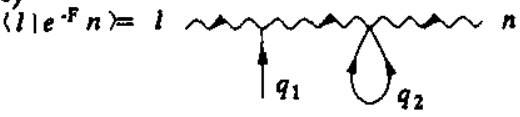

(d)

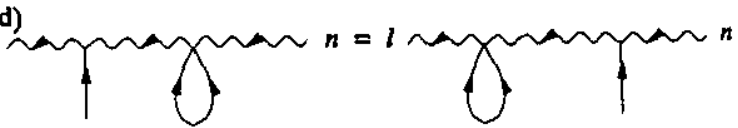

Figure 2. Graphical representations of $(a)(40),(b)(42)$, and (c) the matrix element $\left\langle l\left|e^{-F}\right| n\right\rangle$. (d) Illustration of a diagrammatic proof.

For the wavefunction describing the non-relativistic electron in the large-photonnumber limit we then have

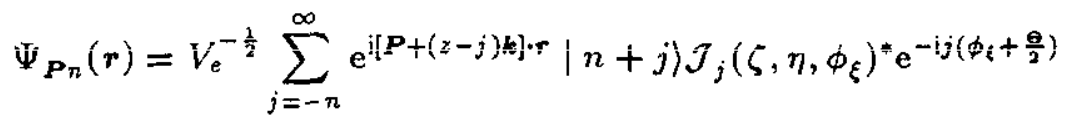

with the energy eigenvalue

$$
\mathcal{E}=\boldsymbol{P}^{2} / 2 m_{e}+\left(n+\frac{1}{2}\right) \omega+z \omega
$$

where

$$
z=\frac{e^{2} \Lambda^{2}}{m_{e} \omega}
$$

These solutions completely agree with the known results of our earlier work (Guo et al 1989, Guo 1990, and I). The 'integration' and the graphical technique are readily generalized for multimode fields, as discussed in the following sections. 
3. Electron in a two-mode strong radiation field

The Hamiltonian for an electron interacting with a two-mode photon field is

$$
\begin{aligned}
H=\frac{(-\mathrm{i} \nabla)^{2}}{2 m_{e}} & -\frac{e}{2 m_{e}}\left[(-\mathrm{i} \nabla) \cdot \boldsymbol{A}_{1}\left(-\boldsymbol{k}_{1} \cdot \boldsymbol{r}\right)+(-\mathrm{i} \nabla) \cdot \boldsymbol{A}_{2}\left(-\boldsymbol{k}_{2} \cdot \boldsymbol{r}\right)\right. \\
& \left.+\boldsymbol{A}_{1}\left(-\boldsymbol{k}_{1} \cdot \ddot{\boldsymbol{r}}\right) \cdot(-\mathrm{i} \nabla)+\boldsymbol{A}_{2}\left(-\boldsymbol{k}_{2} \cdot \boldsymbol{r}\right) \cdot(-\mathrm{i} \nabla)\right] \\
& +\frac{e^{2}\left[\boldsymbol{A}_{1}\left(-\boldsymbol{k}_{1} \cdot \boldsymbol{r}\right)+\boldsymbol{A}_{2}\left(-\boldsymbol{k}_{2} \cdot \boldsymbol{r}\right)\right]^{2}}{2 m_{e}}+\omega_{1} N_{a_{1}}+\omega_{2} N_{a_{2}}
\end{aligned}
$$

where

$$
\begin{aligned}
& A_{i}\left(-k_{i} \cdot r\right)=g_{i}\left(\epsilon_{i} \mathrm{e}^{i k_{i} \cdot r} a_{i}+\epsilon_{i}^{*} \mathrm{e}^{-i k_{i} \cdot r} a_{i}^{\dagger}\right) \quad g_{i}=\left(2 V_{\gamma_{i}} \omega_{i}\right)^{-\frac{1}{2}} \\
& N_{a_{i}}=\frac{1}{2}\left(a_{i} a_{i}^{\dagger}+a_{i}^{\dagger} a_{i}\right) \quad(i=1,2)
\end{aligned}
$$

Generalizing the steps leading to (9), we apply the transformation

$$
\Psi(\boldsymbol{r})=\mathrm{e}^{-\mathrm{i} \boldsymbol{k}_{1} \cdot \boldsymbol{r} N_{\mathrm{a}_{1}}-\mathrm{i} \boldsymbol{k}_{2} \cdot \boldsymbol{r} N_{a_{2}}} \phi(\boldsymbol{r})
$$

and the transformation

$$
\phi(r)=\mathrm{e}^{\mathrm{ip} \cdot \boldsymbol{r} \phi} .
$$

The eigenvalue equation of the Hamiltonian (50) then becomes

$$
\begin{gathered}
\left(\frac{1}{2 m_{e}}\left(p-k_{1} N_{a_{1}}-k_{2} N_{a_{2}}\right)^{2}-\frac{e}{2 m_{e}}\left[\left(\boldsymbol{p}-\boldsymbol{k}_{1} N_{a_{1}}-\boldsymbol{k}_{2} N_{a_{2}}\right) \cdot\left(\boldsymbol{A}_{1}+\boldsymbol{A}_{2}\right)\right.\right. \\
\left.+\left(\boldsymbol{A}_{1}+\boldsymbol{A}_{2}\right) \cdot\left(\boldsymbol{p}-\boldsymbol{k}_{1} N_{a_{1}}-\boldsymbol{k}_{2} N_{a_{2}}\right)\right]+\frac{e^{2}\left(\boldsymbol{A}_{1}+\boldsymbol{A}_{2}\right)^{2}}{2 m_{e}} \\
\left.+\omega_{1} N_{a_{1}}+\omega_{2} N_{a_{2}}\right) \phi=\mathcal{E} \phi
\end{gathered}
$$

where

$$
A_{i}=g_{i}\left(\epsilon_{i} a_{i}+\epsilon_{i}^{*} a_{i}^{\dagger}\right) \quad(i=1,2) .
$$

As in the single-mode case (cf (10)), we now define a vector $\boldsymbol{P}$ such that

$$
P=p-\kappa_{1} k_{1}-\kappa_{2} k_{2}
$$

where $\kappa_{1}$ and $\kappa_{2}$ are $c$-numbers determined by the requirement that the effect of the operator $k_{1} N_{a_{1}}+\bar{k}_{2} \hat{N}_{a_{2}}$ can be replaced by $\kappa_{1} \bar{k}_{1}+\kappa_{2} k_{2}$ in the non-relativistic limit, with $\kappa_{1}$ and $\kappa_{2}$ to be determined later (see (79) below). Then, (54) reduces to

$$
\left(\frac{\boldsymbol{P}^{2}}{2 m_{e}}-\frac{e \boldsymbol{P} \cdot\left(\boldsymbol{A}_{1}+\boldsymbol{A}_{2}\right)}{m_{e}}+\frac{e^{2} \boldsymbol{A}_{1}^{2}}{2 m_{e}}+\frac{e^{2} \boldsymbol{A}_{2}^{2}}{2 m_{e}}+\frac{e^{2} \boldsymbol{A}_{1} \cdot \boldsymbol{A}_{2}}{m_{e}}+\omega_{1} N_{a_{1}}+\omega_{2} N_{a_{2}}\right) \phi=\mathcal{E} \phi .
$$


The polarization vectors satisfy the relations

$$
\begin{aligned}
& \epsilon_{1} \cdot \epsilon_{1}^{*}=\epsilon_{2} \cdot \epsilon_{2}^{*}=1 \quad \epsilon_{i} \cdot \epsilon_{i}=\cos \xi_{i} \mathrm{e}^{i \Theta_{i}} \quad(i=1,2) \\
& \epsilon_{1} \cdot \epsilon_{2}=\cos \left[\frac{1}{2}\left(\xi_{1}+\xi_{2}\right)\right] \mathrm{e}^{i \frac{1}{2}\left(\Theta_{1}+\Theta_{2}\right)} \quad \epsilon_{1} \cdot \epsilon_{2}^{*}=\cos \left[\frac{1}{2}\left(\xi_{1}-\xi_{2}\right)\right] \mathrm{e}^{i \frac{1}{2}\left(\Theta_{1}-\Theta_{2}\right)}
\end{aligned}
$$

and similarly for the complex conjugates. Equation (57) can be rewritten as

$$
\left(\frac{\boldsymbol{P}^{2}}{2 m_{e}}+H_{\gamma}^{\prime}+V^{\prime}\right) \phi=\mathcal{E} \phi
$$

where

$$
\begin{aligned}
& H_{\gamma}^{\prime}=\left(\omega_{1}+\right.\left.e^{2} g_{1}^{2} / m_{e}\right) N_{a_{1}}+\left(\omega_{2}+e^{2} g_{2}^{2} / m_{e}\right) N_{a_{2}} \\
& V^{\prime}=-\frac{e g_{1}}{m_{e}}\left(\boldsymbol{P} \cdot \epsilon_{1} a_{1}+\boldsymbol{P} \cdot \epsilon_{1}^{*} a_{1}^{\dagger}\right)-\frac{e g_{2}}{m_{e}}\left(\boldsymbol{P} \cdot \epsilon_{2} a_{2}+\boldsymbol{P} \cdot \epsilon_{2}^{*} a_{2}^{\dagger}\right) \\
& \quad+\frac{e^{2} g_{1}^{2}}{2 m_{e}}\left(\cos \xi_{1}\right)\left(\mathrm{e}^{\mathrm{i} \Theta_{1}} a_{1}^{2}+\mathrm{e}^{-\mathrm{i} \Theta_{1}} a_{1}^{\dagger 2}\right)+\frac{e^{2} g_{2}^{2}}{2 m_{e}}\left(\cos \xi_{2}\right)\left(\mathrm{e}^{\mathrm{i} \Theta_{2}} a_{2}^{2}+\mathrm{e}^{-\mathrm{i} \Theta_{2}} a_{2}^{\dagger 2}\right) \\
&+\frac{e^{2} g_{1} g_{2}}{m_{e}}\left(\epsilon_{1} \cdot \epsilon_{2} a_{1} a_{2}+\epsilon_{1}^{*} \cdot \epsilon_{2}^{*} a_{1}^{\dagger} a_{2}^{\dagger}\right)+\frac{e^{2} g_{1} g_{2}}{m_{e}}\left(\epsilon_{1} \cdot \epsilon_{2}^{*} a_{1} a_{2}^{\dagger}+\epsilon_{1}^{*} \cdot \epsilon_{2} a_{1}^{\dagger} a_{2}\right) .
\end{aligned}
$$

The 'differentiation' and 'integration' table (19) applies to each mode. Extension of the table for the cross terms for two modes is as follows:

$$
\begin{aligned}
& \left(a_{1} a_{2}\right)=-\left[\omega_{1}+\omega_{2}+e^{2}\left(g_{1}^{2}+g_{2}^{2}\right) / m_{e}\right] a_{1} a_{2} \\
& \left(a_{1}^{\dagger} a_{2}^{\dagger}\right)=\left[\omega_{1}+\omega_{2}+e^{2}\left(g_{1}^{2}+g_{2}^{2}\right) / m_{e}\right] a_{1}^{\dagger} a_{2}^{\dagger} \\
& \left(a_{1} a_{2}^{\dagger}\right)=\left[\omega_{2}-\omega_{1}+e^{2}\left(g_{2}^{2}-g_{1}^{2}\right) / m_{e}\right] a_{1} a_{2}^{\dagger} \\
& \left(a_{1}^{\dagger} a_{2}\right)=\left[\omega_{1}-\omega_{2}+e^{2}\left(g_{1}^{2}-g_{2}^{2}\right) / m_{e}\right] a_{1}^{\dagger} a_{2} \\
& \int\left(a_{1} a_{2}\right)=-\frac{m_{e}}{m_{e}\left(\omega_{1}+\omega_{2}\right)+e^{2}\left(g_{1}^{2}+g_{2}^{2}\right)} a_{1} a_{2} \\
& \int\left(a_{1}^{\dagger} a_{2}^{\dagger}\right)=\frac{m_{e}}{m_{e}\left(\omega_{1}+\omega_{2}\right)+e^{2}\left(g_{1}^{2}+g_{2}^{2}\right)} a_{1}^{\dagger} a_{2}^{\dagger} \\
& \int\left(a_{1} a_{2}^{\dagger}\right)=\frac{m_{e}}{m_{e}\left(\omega_{2}-\omega_{1}\right)+e^{2}\left(g_{2}^{2}-g_{1}^{2}\right)} a_{1} a_{2}^{\dagger} \\
& \int\left(a_{1}^{\dagger} a_{2}\right)=\frac{m_{e}}{m_{e}\left(\omega_{1}-\omega_{2}\right)+e^{2}\left(g_{1}^{2}-g_{2}^{2}\right)} a_{1}^{\dagger} a_{2} .
\end{aligned}
$$


By means of this table, we can 'integrate' $V^{\prime}$ :

$$
\begin{aligned}
& F=\int V^{\prime}=F_{1}+F_{2}+F_{3}+F_{4}+F_{5}+F_{6} \\
& F_{1}=\Delta_{1}^{*} a_{1}-\Delta_{1} a_{1}^{\dagger} \quad \Delta_{1}=\frac{e g_{1}}{m_{e} \omega_{1}+e^{2} g_{1}^{2}} P \cdot \epsilon_{1}^{*} \\
& F_{2}=\Delta_{2}^{*} a_{2}-\Delta_{2} a_{2}^{\dagger} \quad \Delta_{2}=\frac{e g_{2}}{m_{e} \omega_{2}+e^{2} g_{2}^{2}} P \cdot \epsilon_{2}^{*} \\
& F_{3}=\Delta_{3}^{*} a_{1}^{2}-\Delta_{3} a_{1}^{\dagger 2} \quad \Delta_{3}=-\frac{e^{2} g_{1}^{2} \cos \xi_{1}}{4\left(m_{e} \omega_{1}+e^{2} g_{1}^{2}\right)} \mathrm{e}^{-i \Theta_{1}} \\
& F_{4}=\Delta_{4}^{*} a_{2}^{2}-\Delta_{4} a_{2}^{\dagger 2} \quad \Delta_{4}=-\frac{e^{2} g_{2}^{2} \cos \xi_{2}}{4\left(m_{e} \omega_{2}+e^{2} g_{2}^{2}\right)} e^{-i \Theta_{2}} \\
& F_{5}=\Delta_{5}^{*} a_{1} a_{2}-\Delta_{5} a_{1}^{\dagger} a_{2}^{\dagger} \quad \Delta_{5}=-\frac{e^{2} g_{1} g_{2} \epsilon_{1}^{*} \cdot \epsilon_{2}^{*}}{m_{e}\left(\omega_{1}+\omega_{2}\right)+e^{2}\left(g_{1}^{2}+g_{2}^{2}\right)} \\
& F_{6}=\Delta_{6}^{*} a_{1} a_{2}^{\dagger}-\Delta_{6} a_{1}^{\dagger} a_{2} \quad \Delta_{6}=\frac{e^{2} g_{1} g_{2} \epsilon_{1}^{*} \cdot \epsilon_{2}}{m_{e}\left(\omega_{2}-\omega_{1}\right)+e^{2}\left(g_{2}^{2}-g_{1}^{2}\right)}
\end{aligned}
$$

The solution of (59) can be expressed as

$$
\phi=e^{-F}\left|n_{1}, n_{2}\right\rangle=\sum_{l_{1}, l_{2}}\left|l_{1}, l_{2}\right\rangle\left\langle l_{1}, l_{2}\left|e^{-F}\right| n_{1}, n_{2}\right\rangle
$$

where $\left|n_{1}, n_{2}\right\rangle$ is a free-photon state of two modes, $\left|n_{1}, n_{2}\right\rangle=\left|n_{1}\right\rangle\left|n_{2}\right\rangle$. We shall evaluate the matrix element $\left\langle l_{1}, l_{2}\left|e^{-F}\right| n_{1}, n_{2}\right\rangle$ in the large-photon-number limit. Before giving the detailed proof, we indicate the graphical representation for the matrix element and write the algebraic expression according to the graph. We have

$$
\begin{aligned}
\left\langle l_{1}, l_{2}\left|\mathrm{e}^{-F}\right|\right. & \left.n_{1}, n_{2}\right\rangle \\
= & \text { graph shown in figure } 3 \\
= & \sum_{m_{1}, \ldots m_{6}, q_{1}, \ldots, q_{6}} J_{-q_{1}}\left(\zeta_{1}\right) \mathrm{e}^{-\mathrm{i} q_{1} \phi_{1}} J_{-q_{2}}\left(\zeta_{2}\right) \mathrm{e}^{-\mathrm{i} q_{2} \phi_{2}} J_{-q_{3}}\left(\zeta_{3}\right) \mathrm{e}^{-\mathrm{i} q_{3} \phi_{3}} \\
& \times J_{-q_{4}}\left(\zeta_{4}\right) \mathrm{e}^{-\mathrm{i} q_{4} \phi_{4}} J_{-q_{5}}\left(\zeta_{5}\right) \mathrm{e}^{-\mathrm{i} q_{5} \phi_{5}} J_{-q_{6}}\left(\zeta_{6}\right) \mathrm{e}^{-\mathrm{i} q_{6} \phi_{6}} \\
& \times \delta_{l_{1}-m_{1}, q_{1}} \delta_{l_{2}-m_{2}, q_{2}} \delta_{m_{1}-m_{3}, 2 q_{3}} \delta_{m_{2}-m_{4}, 2 q_{4}} \\
& \times \delta_{m_{3}-m_{5}, q_{3}} \delta_{m_{4}-m_{6}, q_{5}} \delta_{m_{5}-n_{1}, q_{6}} \delta_{n_{2}-m_{6}, q_{6}} \\
= & \sum_{q_{1}, \ldots, q_{6}} J_{-q_{2}}\left(\zeta_{1}\right) \mathrm{e}^{-\mathrm{i} q_{1} \phi_{1}} J_{-q_{2}}\left(\zeta_{2}\right) \mathrm{e}^{-\mathrm{i} q_{2} \phi_{2}} \ldots J_{-q_{6}}\left(\zeta_{6}\right) \mathrm{e}^{-\mathrm{i} q_{6} \phi_{6}} \\
& \times \delta_{l_{1}-n_{1}, q_{1}+2 q_{3}+q_{5}+q_{6}} \delta_{l_{2}-n_{2}, q_{2}+2 q_{4}+q_{5}-q_{6}} .
\end{aligned}
$$

By using the formulae developed in section 2 we are able to evaluate the single-mode parts in the diagram of figure 3 , which are due to the factors $e^{-F_{1}}$, $e^{-F_{2}}, e^{-F_{3}}$, and $e^{-F_{4}}$. The arguments $\zeta_{1}, \zeta_{2}, \zeta_{3}$, and $\zeta_{4}$ of the Bessel functions 
$J_{-q_{1}}\left(\zeta_{1}\right), \ldots J_{-q_{1}}\left(\zeta_{4}\right)$ and the phase angles $\phi_{1}, \phi_{2}, \phi_{3}$, and $\phi_{4}$ can immediately be written down in terms of dynamic parameters

$$
\begin{aligned}
\zeta_{1} & =\frac{2|e| \Lambda_{1}}{m_{e} \omega_{1}}\left|\boldsymbol{P} \cdot \epsilon_{1}\right| \quad \phi_{1}=\tan ^{-1}\left[\left(P_{y} / P_{x}\right) \tan \left(\xi_{1} / 2\right)\right]+\frac{1}{2} \Theta_{1} \\
\zeta_{2} & =\frac{2|e| \Lambda_{2}}{m_{e} \omega_{2}}\left|\boldsymbol{P} \cdot \epsilon_{2}\right| \quad \phi_{2}=\tan ^{-1}\left[\left(P_{y} / P_{x}\right) \tan \left(\xi_{2} / 2\right)\right]+\frac{1}{2} \Theta_{2} \\
\zeta_{3} & =\frac{1}{2} z_{1} \cos \xi_{1} \quad \phi_{3}=\Theta_{1} \\
\zeta_{4} & =\frac{1}{2} z_{2} \cos \xi_{2} \quad \phi_{4}=\Theta_{2}
\end{aligned}
$$

where we have applied the limiting process decribed by (32) to each mode, i.e.

$$
g_{i} \sqrt{n_{i}} \rightarrow \Lambda_{i} \quad\left(n_{i} \rightarrow \infty, g_{i} \rightarrow 0\right)
$$

and

$$
z_{i}=\frac{e^{2} \Lambda_{i}^{2}}{m_{e} \omega_{i}} \quad(i=1,2)
$$

Figure 3. Graphical representation of (64).

For the parts due to $e^{-F_{5}}$ and $e^{-F_{6}}$ we need to provide proofs. For the $e^{-F_{5}}$ part, we note first of all that $\Delta_{5} \sqrt{m_{1} m_{2}}$ tends to a finite value in the large-photonnumber limit (cf (62)). The matrix element of the commutator thus tends to zero, if it is not equal to zero:

$$
\begin{aligned}
& \left\langle l_{1}, l_{2}\left|\left[\Delta_{5} a_{1}^{\dagger} a_{2}^{\dagger}, \Delta_{5}^{*} a_{1} a_{2}\right]\right| m_{1}, m_{2}\right\rangle \rightarrow \Delta_{5} \Delta_{5}^{*} m_{2}+\Delta_{5} \Delta_{5}^{*} m_{1} \\
& =\Delta_{5} \Delta_{5}^{*} m_{1} m_{2}\left(m_{1}^{-1}+m_{2}^{-1}\right) \rightarrow 0 \quad\left(m_{1}, m_{2} \rightarrow \infty, g_{1}, g_{2} \rightarrow 0\right) .
\end{aligned}
$$

Thus we have

$$
e^{-F_{5}}=\exp \left(\Delta_{5} a_{1}^{\dagger} a_{2}^{\dagger}-\Delta_{5}^{*} a_{1} a_{2}\right) \rightarrow \sum_{s, q} \frac{\left(\Delta_{5} a_{1}^{\dagger} a_{2}^{\dagger}\right)^{s}\left(\Delta_{5} a_{1}^{\dagger} a_{2}^{\dagger}\right)^{q}\left(-\Delta_{5}^{*} a_{1} a_{2}\right)^{q}}{(s+q) ! q !}
$$


and

$$
\begin{gathered}
\left\langle l_{1}, l_{2}\left|e^{-F_{s}}\right| m_{1}, m_{2}\right\rangle \rightarrow \sum_{s=-\infty}^{\infty} J_{-s}\left(\zeta_{5}\right) \exp \left(-i s \phi_{5}\right) \delta_{l_{1}-m_{1}, s} \delta_{l_{2}-m_{2}, s} \\
\left(m_{1}, m_{2}, l_{1}, l_{2} \rightarrow \infty, g_{1}, g_{2} \rightarrow 0\right)
\end{gathered}
$$

which equals the diagram in figure $4(a)$. The arguments are found to be

$$
\begin{aligned}
& \Delta_{5} \sqrt{m_{1} m_{2}} \rightarrow-\frac{1}{2} \zeta_{5} \mathrm{e}^{-i \phi_{5}} \\
& \zeta_{5}=2 \lim _{m_{1}, m_{2} \rightarrow \infty ; g_{1}, g_{2} \rightarrow 0}\left|\Delta_{5} \sqrt{m_{1} m_{2}}\right| \\
& \quad=2 e^{2} \Lambda_{1} \Lambda_{2} \cos \left[\frac{1}{2}\left(\xi_{1}+\xi_{2}\right)\right] /\left(\omega_{1}+\omega_{2}\right) m_{e} \\
& \phi_{5}=-\arg \left(-\Delta_{5} \sqrt{m_{1} m_{2}}\right)=\frac{1}{2}\left(\Theta_{1}+\Theta_{2}\right) .
\end{aligned}
$$

(a)

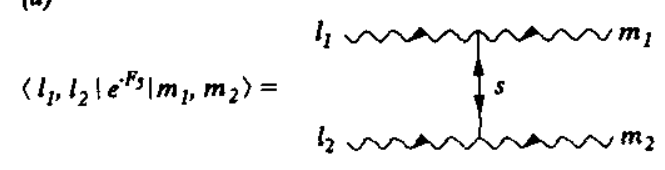

(b) $\left\langle m_{1}, m_{2}\right| e^{-F_{\theta}\left|n_{1}, n_{2}\right\rangle=n_{1}}$

Figure 4. Graphical representation of (a) (70) and (b) (73).

The $e^{-F_{6}}$ part can be obtained in a similar way. We can expand $e^{-F_{6}}$ as

$$
e^{-F_{6}} \rightarrow \sum_{s, q} \frac{\left(\Delta_{6} a_{1}^{\dagger} a_{2}\right)^{s}\left(\Delta_{6} a_{1}^{\dagger} a_{2}\right)^{q}\left(-\Delta_{6}^{*} a_{1} a_{2} \dagger\right)^{q}}{(s+q) ! q !}
$$

Thus, we have

$$
\begin{aligned}
\left\langle m_{1}, m_{2}\left|e^{-F_{6}}\right| n_{1}, n_{2}\right\rangle \rightarrow \sum_{s=-\infty}^{\infty} J_{-s}\left(\zeta_{6}\right) \exp \left(-\mathrm{i} s \phi_{6}\right) \delta_{m_{1}-n_{1}, s} \delta_{n_{2}-m_{2}, s} \\
\quad\left(n_{1}, n_{2}, m_{1}, m_{2} \rightarrow \infty, g_{1}, g_{2} \rightarrow 0\right) \\
=\text { diagram in figure } 4(b) .
\end{aligned}
$$


The arguments are found to be

$$
\begin{aligned}
& \Delta_{6} \sqrt{n_{1} n_{2}} \rightarrow-\frac{1}{2} \zeta_{6} \mathrm{e}^{-\mathrm{i} \phi_{6}} \\
& \zeta_{6}=2 \lim _{n_{1}, n_{2} \rightarrow \infty ; g_{1}, g_{2} \rightarrow 0}\left|\Delta_{6} \sqrt{n_{1} n_{2}}\right| \\
& \quad=2 e^{2} \Lambda_{1} \Lambda_{2} \cos \left[\frac{1}{2}\left(\xi_{1}-\xi_{2}\right)\right] /\left|\omega_{2}-\omega_{1}\right| m_{e} \\
& \phi_{6}=-\arg \left(-\Delta_{6} \sqrt{n_{1} n_{2}}\right)= \begin{cases}\frac{1}{2}\left(\Theta_{1}-\Theta_{2}\right) & \omega_{1}>\omega_{2} \\
\frac{1}{2}\left(\Theta_{1}-\Theta_{2}\right)-\pi & \omega_{1}<\omega_{2} .\end{cases}
\end{aligned}
$$

This completes the proof of the graphical representation of the two-mode case.

Under the transformation $e^{F}$, (59) becomes

$$
\left(\boldsymbol{P}^{2} / 2 m_{e}+H_{\gamma}^{\prime}\right)\left(e^{F} \phi\right)=\mathcal{E}\left(e^{F} \phi\right)
$$

Introducing the solution (63) into this equation, the energy eigenvalue can be evaluated in the large-photon-number limit as

$$
\mathcal{E}=\boldsymbol{P}^{2} / 2 m_{e}+\left(n_{1}+\frac{1}{2}+z_{1}\right) \omega_{1}+\left(n_{2}+\frac{1}{2}+z_{2}\right) \omega_{2} .
$$

By setting

$$
E=\frac{\boldsymbol{P}^{2}}{2 m_{e}}
$$

we obtain a covariant expression

$$
\mathcal{E}+m_{e}=\left(E+m_{e}\right)+\kappa_{1} \omega_{1}+\kappa_{2} \omega_{2} \quad \boldsymbol{P}=\boldsymbol{p}-\kappa_{1} \boldsymbol{k}_{1}-\kappa_{2} \boldsymbol{k}_{2}
$$

where

$$
\kappa_{1}=n_{1}+\frac{1}{2}+z_{1} \quad \kappa_{2}=n_{2}+\frac{1}{2}+z_{2} .
$$

It can be verified, as in the single-mode case, that these values are correct at least up to leading order in $v / c$.

Just as in the single-mode case, we can define a 'generalized Bessel function' $\mathcal{J}_{j_{1} j_{2}}(\zeta)$, where $\zeta=\left(\zeta_{1}, \zeta_{2}, \ldots, \zeta_{6}\right)$, such that in the large-photon-number limit

$$
\begin{gathered}
\left\langle l_{1}, l_{2}\left|\mathrm{e}^{-F}\right| n_{1}, n_{2}\right\rangle=\mathrm{e}^{-\mathrm{i}\left(l_{1}-n_{1}\right) \phi_{1}} \mathrm{e}^{-\mathrm{i}\left(l_{2}-n_{2}\right) \phi_{2}} \mathcal{J}_{l_{1}-n_{1}, l_{2}-n_{2}}(\zeta)^{*} \\
=\sum_{j_{1}, j_{2}} \mathrm{e}^{-\mathrm{i} j_{1} \phi_{1}} \mathrm{e}^{-\mathrm{i} j_{2} \phi_{2}} \mathcal{J}_{j_{1} j_{2}}(\zeta)^{*} \delta_{l_{1}-n_{1}, j_{1}} \delta_{l_{3}-n_{2}, j_{2}} .
\end{gathered}
$$

From (64), the two-mode generalized Bessel function is

$$
\begin{aligned}
\mathcal{J}_{j_{1} j_{2}}(\zeta)= & \sum_{q_{3}, q_{s}, q_{5}, q_{6}} J_{-j_{1}+2 q_{3}+q_{5}+q_{s}}\left(\zeta_{1}\right) \mathrm{e}^{-\mathrm{i}\left(2 q_{3}+q_{5}+q_{8}\right) \phi_{1}} \\
& \times J_{-j_{2}+2 q_{4}+q_{5}-q_{8}}\left(\zeta_{2}\right) \mathrm{e}^{-\mathrm{i}\left(2 q_{4}+q_{5}-q_{6}\right) \phi_{2}} J_{-q_{s}}\left(\zeta_{3}\right) \mathrm{e}^{\mathrm{i} \varphi_{5} \phi_{3}} \ldots J_{-q_{s}}\left(\zeta_{6}\right) \mathrm{e}^{\mathrm{i} q_{8} \phi_{8}} .
\end{aligned}
$$


With this notation, the coordinate-independent solution (63) can be expressed as

$$
\phi=\sum_{j_{1}=-n_{1}, j_{2}=-n_{2}}^{\infty} \mathcal{J}_{j_{1} j_{2}}(\zeta)^{*} \mathrm{e}^{-\mathrm{i}\left(j_{1} \phi_{1}+j_{2} \phi_{2}\right)}\left|n_{1}+j_{1}, n_{2}+j_{2}\right\rangle .
$$

The final form of the two-mode wavefunction is

$$
\begin{aligned}
\Psi_{P n_{1}, n_{2}}(\boldsymbol{r})= & V_{e^{-\frac{1}{2}}} \mathrm{e}^{\mathrm{i}\left(\boldsymbol{P}+z_{1} \boldsymbol{k}_{1}+z_{2} \boldsymbol{k}_{2}\right) \cdot \boldsymbol{r}} \\
& \times \sum_{j_{1}=-n_{1}, j_{2}=-n_{2}}^{\infty} \mathcal{J}_{j_{1} j_{2}}(\zeta)^{*} \mathrm{e}^{-\mathrm{i}\left[j_{1}\left(\boldsymbol{k}_{1} \cdot \boldsymbol{r}+\phi_{1}\right)+j_{2}\left(\boldsymbol{k}_{2} \cdot \boldsymbol{r}+\phi_{2}\right)\right]}\left|n_{1}+j_{1}, n_{2}+j_{2}\right\rangle
\end{aligned}
$$

with the energy levels given by (76).

\section{Generalization}

The technique developed above can be generalized to an arbitrary number of modes. To do so, we introduce a vector space $M$. A vector in this space is denoted by a letter with an overbar; the index of the components of the vector runs over all modes of the quantized radiation field. In the three-mode case, for example, the vectors are $\bar{z}=\left(z_{1}, z_{2}, z_{3}\right), \bar{k}=\left(k_{1}, k_{2}, k_{3}\right)$, etc. In general, we have

$$
\bar{z}=\left(z_{1}, z_{2}, \ldots\right) \quad \vec{k}=\left(k_{1}, k_{2}, \ldots\right) .
$$

The inner product is denoted by o, e.g.

$$
\ddot{z} \circ \bar{k}=z_{1} k_{1}+z_{2} k_{2}+\cdots \text {. }
$$

In this notation, the Hamiltonian for the multimode case is

$$
H=\frac{(-\mathrm{i} \nabla)^{2}}{2 m_{e}}-\frac{e}{2 m_{e}}[(-\mathrm{i} \nabla) \cdot \boldsymbol{A}(-\bar{k} \cdot \boldsymbol{r})+\boldsymbol{A}(-\bar{k} \cdot \boldsymbol{r}) \cdot(-\mathrm{i} \nabla)]+\frac{e^{2} \boldsymbol{A}^{2}(-\bar{k} \cdot \boldsymbol{r})}{2 m_{e}}+\bar{\omega} \circ \bar{N}_{\bar{d}}
$$

where

$$
\boldsymbol{A}(-\bar{k} \cdot \boldsymbol{r})=\boldsymbol{A}_{1}\left(-\boldsymbol{k}_{1} \cdot \boldsymbol{r}\right)+\boldsymbol{A}_{2}\left(-\boldsymbol{k}_{2} \cdot \boldsymbol{r}\right)+\cdots \quad \bar{N}_{\bar{a}}=\left(N_{a_{1}}, N_{a_{2}}, \ldots\right) .
$$

The eigenfunctions of the Hamiltonian (86) as solutions of the Schrödinger equation in the large-photon-number limit are

$$
\Psi_{\boldsymbol{P} \bar{n}}(\boldsymbol{r})=V_{e}^{-\frac{1}{2}} \mathrm{e}^{\mathrm{i}[(\boldsymbol{P}+\bar{z} 0 \overline{\boldsymbol{k}}) \cdot \boldsymbol{r}]} \sum_{j=-\bar{n}}^{\infty} \mathcal{J}_{j}(\zeta)^{*} \mathrm{e}^{-\mathrm{i}[\bar{j} 0(\overline{\boldsymbol{k}} \cdot \boldsymbol{r}+\bar{\phi})]}|\bar{n}+\bar{j}\rangle
$$

where

$$
\zeta=\left(\zeta_{1}, \zeta_{2}, \ldots, \zeta_{m(m+1)}\right)
$$


and $m$ is the number of modes. The corresponding energy eigenvalues are

$$
\mathcal{E}=\boldsymbol{P}^{2} / 2 m_{e}+\left(\bar{n}+\frac{\overline{1}}{2}\right) \circ \bar{\omega}+\bar{z} \circ \bar{\omega}
$$

where $\frac{1}{2}$ is understood as a vector $\left(\frac{1}{2}, \frac{1}{2}, \ldots\right)$.

For $m$ modes, the generalized Bessel functions $\mathcal{J}_{j}(\zeta)$ is composed of $m(m+1)$ fold ordinary Bessel functions. They are defined through the matrix elements

$$
\left\langle\bar{l}\left|e^{-F}\right| \bar{n}\right\rangle=\sum_{\bar{j}} \mathcal{J}_{\bar{j}}(\zeta)^{*} \mathrm{e}^{-\mathrm{i} j \overline{ } 0 \bar{\phi}} \delta_{\bar{l}-\bar{n}, \bar{j}}
$$

and $\left\langle\bar{l}\left|e^{-F}\right| \bar{n}\right\rangle$ is evaluated by the diagram in figure 5 . The arguments of the single Bessel functions and the related phases due to each single-mode interaction can be written down according to the single-mode formulae (40)-(43). Those due to each two-mode interaction, i.e. the cross terms in the interaction, can be written according to the two-mode formulae (70)-(74). In the multimode cases, no types of interactions occur that are different from those in the two-mode case. The multimode interactions have thus been completely solved for non-relativistic and large-photonnumber conditions.

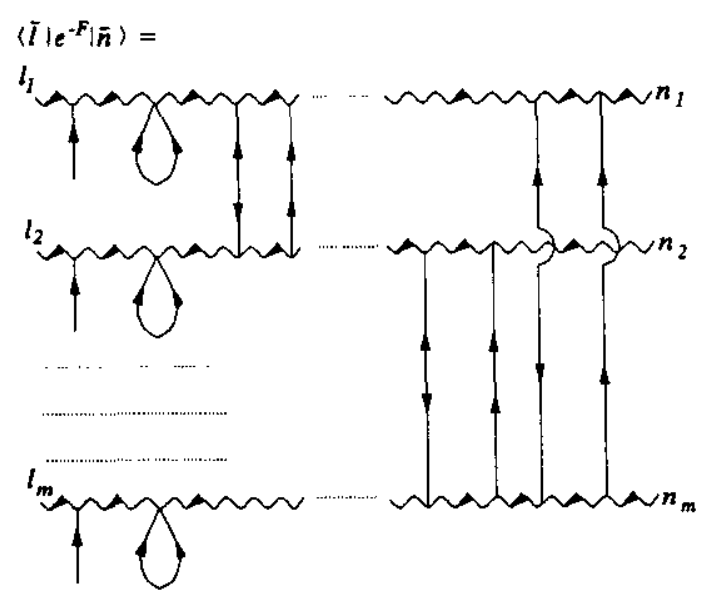

Figure 5. Graphical representation of (91). The graph is invariant under any permutation of the rows.

\section{Discussions}

\subsection{Photon-mode conversion}

The multimode solutions we obtained here offer a powerful means to treat photonmode conversions. Photon-mode conversions appear in many phenomena such as multiphoton ionization, Compton scattering, generation of higher-order harmonics, and the Kapitza-Dirac effect (Kapitza and Dirac 1933, Bucksbaum et al 1988). The multimode solutions can have wide applications to these important effects. Our recent work (Guo and Drake 1992b) shows that in standing-wave multiphoton ionization processes, the photoelectron can absorb photons from one propagating mode and emit into the other propagating mode, thereby explaining the angular distribution peak-splitting (Bucksbaum et al 1988). Our theoretical results show good agreement with experiments. 


\subsection{Generalization of the Keldysh-Faisal-Reiss formula}

As an example of applications, one can use a multimode solution as the final state for a photoelectron to calculate the transition rate in multiphoton ionization processes according to Keldysh-Faisal-Reiss (KFR) theory (Keldysh 1964, Faisal 1973, Reiss 1980). Setting aside questions of validity discussed in I, the KFR theory provides a simple example of how to use the technique developed here to generalize a transition rate formula from the single-mode case to the multimode case.

The KFR multiphoton ionization rate for two laser beams can be easily formulated by using the solution (83) as the final state. A procedure similar to the single-mode case yields the transition rate formula

$$
\begin{gathered}
\frac{\mathrm{d} w}{\mathrm{~d} \Omega}=\frac{\left(2 m_{e}^{3}\right)^{\frac{1}{2}}}{(2 \pi)^{2}}\left(j_{1} \omega_{1}+j_{2} \omega_{2}-z_{1} \omega_{1}-z_{2} \omega_{2}\right)^{2}\left(j_{1} \omega_{1}+j_{2} \omega_{2}-z_{1} \omega_{1}-z_{2} \omega_{2}-E_{\mathrm{b}}\right)^{\frac{1}{2}} \\
\times\left|\Phi\left(P+z_{1} k_{1}+z_{2} k_{2}-j_{1} k_{1}-j_{2} k_{2}\right)\right|^{2}\left|\mathcal{J}_{j_{1} j_{2}}(\zeta)\right|^{2}
\end{gathered}
$$

where $j_{1}$ and $j_{2}$ are absorbed-photon numbers in each mode, and $E_{\mathrm{b}}$ is the binding energy of the initial atomic bound state. In (92) the final electron momentum $\boldsymbol{P}$ is restricted by energy conservation

$$
\frac{P^{2}}{2 m_{e}}=j_{1} \omega_{1}+j_{2} \omega_{2}-z_{1} \omega_{1}-z_{2} \omega_{2}-E_{\mathrm{b}}
$$

\section{Acknowledgments}

D-S Guo would like to thank Bernd Crasemann for his advice, support and help with the manuscript, and Teijo Åberg for stimulating discussions. This work has been supported in part by Natural Science and Engineering Research Council of Canada, the Killam Foundation, and National Science Foundation grant PHY-8908 124.

\section{References}

Bucksbaum P H, Schumacher D W and Bashkansky M 1988 Phys. Rev. Lett. 611162

Chu S-I and Cooper J 1985 Phys. Leth 32A 2769

Ehlotzky F 1985 Can. J. Phys. 63907

Faisal F H M 1973 J. Phys B: At. Mol Opt. Phys. 6 L89

Gordon W 1926 Z. Phys. 40117

Guo D-S 1990 Phys. Lett. 42A 4302

Guo D-S and Asterg T 1988 J. Phys. A: Math. Gen. 214577

Guo D-S, Aberg T and Crasemann B 1989 Phys. Lett. 40A 4997

Guo D-S and Drake G W F 1992a J. Phys. A: Math. Gen. 253383 1992b Phys. Lett. 45A 6622

Kapitza P L and Dirac P A M 1933 Proc. Camb. Phil. Soc. 29297

Keldysh L V 1964 Zh. Eksp. Teor. Fiz 471945 (Engl. transl. 1965 Sov. Phys.-JETP 20 1307)

Loudon R and Knight P L 1987 J. Mod. Opt. 34709

Reiss H R 1980 Phys. Leth. 22A 1786

Rosenberg L 1982 Adv. At. Mol Phys. 181

Volkov D M 1935 Z Phys. 94250 